\title{
Modeling carrier density dependent charge transport in semiconducting carbon nanotube networks
}

\author{
Stefan P. Schieß1, ${ }^{1}$ Xander de Vries, ${ }^{2}$ Marcel Rother, ${ }^{1}$ Andrea Massé, ${ }^{2}$ Maximilian Brohmann, ${ }^{1}$ \\ Peter A. Bobbert, ${ }^{2}$ and Jana Zaumseil ${ }^{1, *}$ \\ ${ }^{1}$ Institute for Physical Chemistry, Universität Heidelberg, Im Neuenheimer Feld 253, D-69120 Heidelberg, Germany \\ ${ }^{2}$ Department of Applied Physics, Technische Universiteit Eindhoven, P.O. Box 513, NL-5600 MB Eindhoven, The Netherlands
}

(Received 11 June 2017; published 27 September 2017)

\begin{abstract}
Charge transport in a network of only semiconducting single-walled carbon nanotubes is modeled as a random-resistor network of tube-tube junctions. Solving Kirchhoff's current law with a numerical solver and taking into account the one-dimensional density of states of the nanotubes enables the evaluation of carrier density dependent charge transport properties such as network mobility, local power dissipation, and current distribution. The model allows us to simulate and investigate mixed networks that contain semiconducting nanotubes with different diameters, and thus different band gaps and conduction band edge energies. The obtained results are in good agreement with available experimental data.
\end{abstract}

DOI: 10.1103/PhysRevMaterials.1.046003

\section{INTRODUCTION}

Networks of single-walled carbon nanotubes (SWNTs) exhibit very high charge carrier mobilities [1-5] while also being flexible and stretchable [6,7]. They can be processed from solution at low temperatures, which makes them attractive candidates for printed and flexible electronics [8,9]. SWNTs are characterized by their chirality vector $(n, m)$, which describes the direction of rolling up a virtual graphene sheet to form a nanotube and the resulting diameter [10]. The chirality vector (or 'chirality') of a nanotube determines its electronic properties, such as being metallic or semiconducting, the band gap of semiconducting SWNTs, and their distinct onedimensional density of states (DOS). As-grown SWNT raw material contains about one third metallic nanotubes, which cause high off currents and limited current modulation in fieldeffect transistors with dense SWNT networks. However, in line of recent advances toward the separation of semiconducting from metallic SWNTs [11-15], it is now possible to obtain semiconducting SWNT networks with purities of more than $99.9 \%$. This progress has enabled the fabrication of field-effect transistors with excellent performance parameters, such as high on/off current ratios and on-conductances [3,16,17]. The availability of purely semiconducting SWNT networks also raises new questions about the impact of network properties on charge transport [18-21]. While it is possible to remove almost all of the metallic nanotubes from a mixture of SWNTs, the remainder often consists of many different semiconducting chiralities with different diameters and thus band gaps. The precise influence of network composition in terms of SWNT diameter distribution is still unclear but has implications for the device performance of field-effect transistors, as recent experimental data show [22].

While charge transport in a single, pristine nanotube is well understood, a universal description of transport in SWNT networks and especially mixed semiconducting networks is still lacking. Temperature-dependent mobility and conductivity measurements on dense SWNT networks clearly show thermally activated transport that might be

\footnotetext{
*zaumseil@uni-heidelberg.de
}

described by the extended variable-range hopping (VRH) or fluctuation-induced tunneling (FIT) model [23-25]. Although fitting mobility data to these models does not provide a conclusion on the microscopic transport mechanism, they suggest that SWNT networks can be treated as disordered percolating systems [26-34].

Several experimental studies have shown the influence of nanotube length and network density on device performance $[18,19]$. Others investigated charge transport on a microscopic scale with, for example, conductive atomic force microscopy (c-AFM), and focused especially on segment and junction resistances. They found values of around $10 \mathrm{k} \Omega \mu \mathrm{m}^{-1}$ for nanotube segments and $10^{2}-10^{5} \mathrm{k} \Omega$ for junctions [29,35-40]. Most of these experiments investigated the role of metallic nanotubes within networks and the highly resistive Schottky barriers between metallic and semiconducting nanotubes. Only a couple of studies reported basic doping dependent measurements of junction resistances in networks and found lower resistances with higher carrier densities [39,40].

From a theory perspective, SWNT networks have been modeled as two-dimensional [26,29,31,32], two-dimensional layered [27,28], or three-dimensional [34] networks of conducting sticks or hollow cylinders. The overall conductivity of these networks was calculated via Kirchhoff's law or with circuit simulation tools, such as SPICE [31,32,34], in which nanotube segments were described as resistors and junctions as resistors and/or diodes (e.g., for Schottky barriers). The analysis generally focused on geometric network parameters, such as SWNT length distribution, nanotube density, metallicto-semiconducting SWNT ratio, and alignment angles. Similar to most experimental studies, no charge carrier density dependent analysis was applied. Instead fixed resistance values for the different components of the network (segments versus junctions, metallic versus semiconducting nanotubes) were assigned. Only one recent study has taken the intrinsic properties of SWNTs, such as the DOS, into account to determine segment resistances in a network [34].

For the application of semiconducting SWNT networks in field-effect transistors the charge carrier density dependent transport properties are crucial, as the carrier density is varied over orders of magnitude from the off to the on state via the gate voltage. Given that the properties of a nanotube network 
are no longer determined by residual metallic nanotubes, the composition of mixed semiconducting SWNTs with different diameters and band gaps becomes important. We have recently shown via electroluminescence spectra of light-emitting fieldeffect transistors based on mixed semiconducting SWNT networks that charge transport depends strongly on carrier density and the specific network composition [22]. The intrinsic DOS of individual SWNT chiralities may not only affect the transport within the nanotubes [34], but also the charge transfer across SWNT junctions, which is often considered to be the limiting transport step. A charge carrier density and chirality dependent description of junction resistances and overall mobilities in networks of semiconducting SWNTs is thus highly desirable.

Here, we address these issues, by treating a thin SWNT layer as a random network of one-dimensional sticks in a two-dimensional periodic box, based on models and solutions for disordered systems [41,42], such as organic semiconductors $[43,44]$. Our main goal is to develop a model that allows us to simulate the charge carrier density dependent transport properties (mobility, local power dissipation, current distribution) of mixed networks of semiconducting SWNTs with different length distributions, energetic disorder and, most importantly, different chiralities and thus DOS. In Sec. II we introduce a model that describes the SWNT layer as a network of random resistors of tube-tube junctions, taking into account the absolute DOS of each nanotube. In Sec. III we will discuss the basic results of the model and the impact of various network parameters, such as the magnitude of the energetic disorder, the SWNT length, and the SWNT density, on transport properties. In Sec. IV we visualize the current flow and power dissipation in these networks, before we compare our simulation results to experimental data in Sec. V, demonstrating the applicability of our approach to realistic mixed networks.

\section{MOBILITY MODEL}

\section{A. Charge transport modeling}

The charge transport within a SWNT network can be divided into two types: (i) transport along an individual SWNT and (ii) transport across SWNT-SWNT junctions. Single nanotubes exhibit carrier mobilities of 1000 to $10000 \mathrm{~cm}^{2} \mathrm{~V}^{-1} \mathrm{~s}^{-1}$ at room temperature and even ballistic transport over distances up to a micrometer $[36,45]$. Hence, we can assume that the limiting step in a dense network is the charge transfer across the junctions. This assumption is corroborated by experimental current-voltage measurements along single nanotubes and across SWNT-SWNT junctions [35,39,40]. Thus, we will neglect the resistance of the SWNT segments between the junctions and assume that charges move instantaneously between two junctions along a given SWNT segment. With this assumption, the nanotube network becomes a network of SWNT-SWNT junctions (see Fig. 1). Since SWNT networks experimentally show thermally activated transport similar to other disordered systems [24,25], we decided to describe the charge transfer across those junctions as a hopping process. We apply a master equation approach, resulting in a random-resistor network valid at low electrical field, where the junctions are modeled as bond conductances $G_{i j}$ between single nanotubes acting as sites/nodes [41-44].

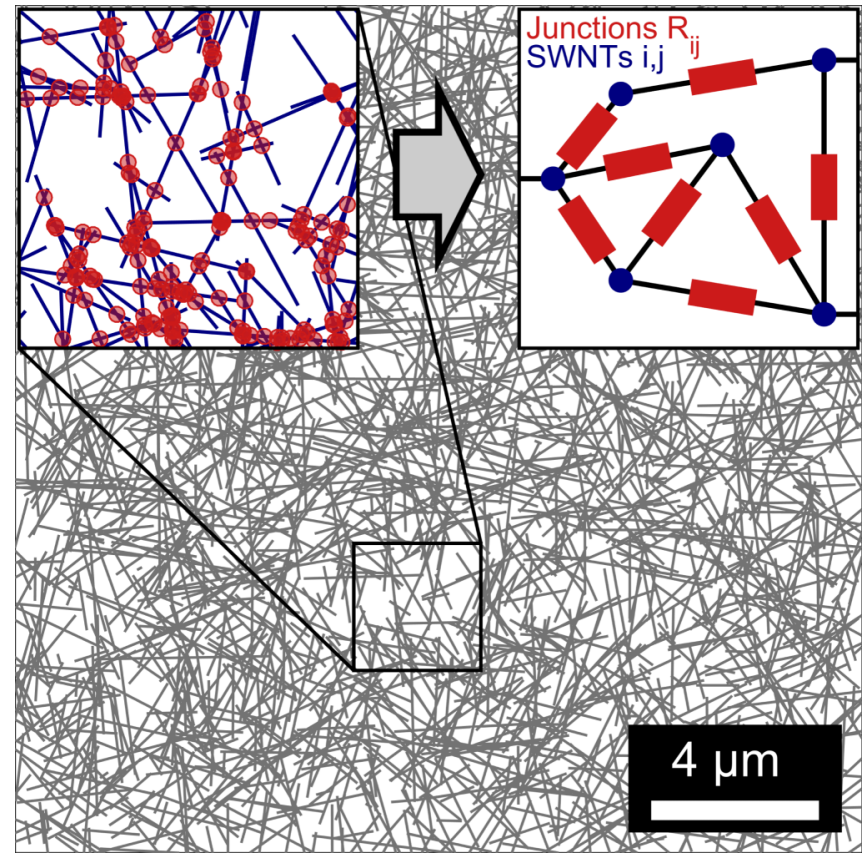

FIG. 1. 2D Network of 1D sticks as a geometric model for a random SWNT network (gray). The magnified inset (left) illustrates the junctions (red) between single nanotubes (blue) and their conversion into a random resistor network (right inset) with junctions acting as resistors and SWNTs as nodes.

The bond conductance between two sites in a disordered system of sites, over which charges can hop, in the case of a small electric field $F$ and in a mean field approximation, is generally given by [44]

$$
G_{i j}\left(E_{F}, E_{i}, E_{j}\right)=\frac{e^{2} \cdot \omega_{i j}^{s y m m}}{4 k_{B} T \cdot \cosh \left(\frac{E_{i}-E_{F}}{2 k_{B} T}\right) \cdot \cosh \left(\frac{E_{j}-E_{F}}{2 k_{B} T}\right)}
$$

with $E_{F}$ the Fermi energy, $e$ the elemental charge, $k_{B}$ the Boltzmann constant, $T$ the absolute temperature, $\omega_{i j}^{\text {symm a }}$ symmetrized hopping rate between sites $i$ and $j$, and $E_{i}$ and $E_{j}$ the local energies of a charge residing on sites $i$ and $j$. The conductance of the system can then be calculated by constructing the random-resistor network of these bond conductances and solving Kirchhoff's equations. This classical approach has to be adjusted in order to account for two major differences between SWNT networks and conventional disordered systems:

(i) In conventional disordered systems (e.g., organic semiconductors), the Coulomb charging energy for two or more charges residing on the same site is so high that double or higher occupancy can be neglected. However, SWNTs have a very low Coulomb charging energy of around $0.9 \mathrm{meV}_{\mu \mathrm{m}}^{-1}$ [46], so that (at room temperature) several charges can reside on one SWNT.

(ii) In conventional disordered systems, a single energy $E_{i}$ is assigned to each site $i$. A Gaussian or exponential distribution is typically attributed to those site energies to account for the intrinsic energetic disorder. A single SWNT, however, does not have a single energy level but rather an intrinsic DOS with van Hove singularities at the bottom of each subband (see Fig. 2). 


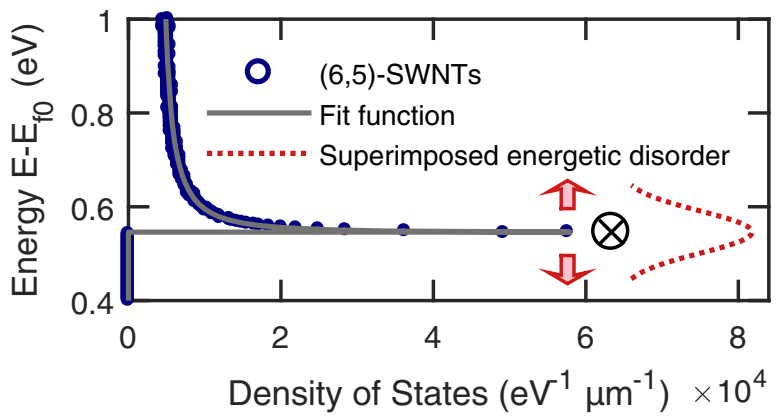

FIG. 2. Density of states (first van Hove singularity, first conduction subband) of a single $(6,5)$ SWNT as calculated within the tight binding approximation including trigonal warping effects (circles). $[47,48]$ The fitted first van Hove singularity (gray line) is used for modeling the SWNT network. To include energetic disorder, the band edge of each SWNT is shifted by a random energy drawn from a Gaussian distribution.

In order to account for the differences (i) and (ii) we treat the states in each SWNT as sites with an energy distribution given by its DOS. The Pauli principle forbids double occupancy of a state in each SWNT in the same way as the high Coulomb energy forbids double occupancy of a site in a conventional disordered system. Neglecting the charging energy of the SWNTs, we can introduce a conductance $G_{i j}^{j u n}$ between two SWNTs $i$ and $j$ connected via a junction $i j$ by modifying equation (1) to

$$
G_{i j}^{j u n}\left(E_{F}\right)=\iint G_{i j}\left(E_{F}, E_{i}, E_{j}\right) \cdot D_{i}\left(E_{i}\right) \cdot D_{j}\left(E_{j}\right) \mathrm{d} E_{i} \mathrm{~d} E_{j},
$$

where $D_{i}(E)$ and $D_{j}(E)$ are the DOS of SWNT $i$ and $j$, respectively, and $G_{i j}\left(E_{F}, E_{i}, E_{j}\right)$ is given by equation (1), where $\omega_{i j}^{\text {symm }}$ is now a symmetrized hopping rate between states with energy $E_{i}$ and $E_{j}$ in SWNT $i$ and $j$, respectively. As we have no specific knowledge about the details of charge transfer between states in the two nanotubes, we use a simplified Miller-Abrahams approach [41], leading to $\omega_{i j}^{\text {symm }}=\omega_{0} \cdot \exp \left(-\left|\Delta E_{i j}\right| / 2 k_{B} T\right)$ [44], with $\omega_{0}$ a hopping prefactor determined by the charge-transfer integral between the states and $\Delta E_{i j}$ the energy difference $E_{j}-E_{i}$ between the states $j$ and $i$. The introduction of a range of different SWNTs with different band gaps and DOS is thus straightforward.

Using the above expression for $G_{i j}^{j u n}$, we can consider an arbitrary network of SWNTs, solve the coupled Kirchhoff's equations for current conservation at each node using a numerical solver, and obtain the network current. From this, the equivalent conductance of the network and therefore the mobility of charge carriers in the network can be calculated.

\section{B. Computational solution}

We first generate a random network of one-dimensional interpenetrating sticks representing the SWNTs with a preset density in a two-dimensional box (dimensions $L_{x}$ and $L_{y}$ ) via the Monte Carlo technique (see Fig. 1). Thus, random, uniformly distributed coordinates for the center of mass and angles for the direction of each SWNT are generated within the simulation box. Treating the network as a two-dimensional system is appropriate for two reasons: (i) Up to a certain surface coverage SWNT networks are quasi two-dimensional except for the junctions. (ii) A two-dimensional approximation is suitable for describing transport in SWNT field-effect transistors, because the charge accumulation layer is only a few nanometers thick.

Subsequently, a certain length and chirality $(n, m)$ are assigned to each SWNT according to predetermined distributions. In order to avoid problems with the periodic boundary conditions (see below), the restriction that SWNTs neither cross the line $x=L_{x}$ nor $y=L_{y}$, nor both is imposed. Both length and chirality are used to calculate the absolute DOS of each SWNT. We then shift the conduction band edge by an energetic disorder value, randomly generated from a Gaussian distribution (see red dotted line, Fig. 2). For simplicity, we only take into account the first subband (first van Hove singularity) of the conduction band and neglect higher-lying subbands. This is a realistic assumption for typical experimental charge carrier densities in field-effect transistors [3].

Then, the junctions between intersecting SWNTs (see Fig. 1, left inset) are determined. In order to reduce boundary effects and obtain a rapid convergence with increasing box size, we replicate the box in all directions by applying periodic boundary conditions. The bond conductances between all SWNTs $i$ and $j$ (across the junction $i j$ ) are calculated using equation (2).

Next, we solve Kirchhoff's equations, taking into account the periodic boundary conditions:

$$
\sum_{i \neq j} G_{i j}^{j u n} \cdot\left(V_{i}-V_{j}+\Delta_{i j}\right)=0,
$$

with

$$
\Delta_{i j}=\left\{\begin{array}{lrl}
F \cdot L_{x}, & x_{j} & >L_{x} \\
0, & & 0 \leqslant x_{j} \leqslant L_{x} \\
-F \cdot L_{x}, & x_{j} & <0
\end{array}\right.
$$

where $F$ is the external electric field, applied in the $x$ direction, $V_{i}$ the local electrostatic potential at SWNT $i, L_{x}$ the length of the simulation box in the $x$ direction, and $x_{j}$ the $x$ position of tube $j$, defined as the position of its center of mass. The biconjugate sparse matrix solver within MATLAB [49] is used to calculate the local potentials $V_{i}$ for different Fermi energies and thus carrier densities. The latter are calculated from the Fermi energies using the Fermi function $f\left(E_{i}, E_{F}\right)$,

$$
n\left(E_{F}\right)=\frac{1}{L_{x} \cdot L_{y}} \sum_{i=0}^{N} \int_{-\infty}^{\infty} D_{i}\left(E_{i}\right) \cdot f\left(E_{i}, E_{F}\right) \mathrm{d} E_{i}
$$

with $L_{y}$ the length of the simulation box in the $y$ direction and $N$ the total number of sites within the box.

Finally, the desired network properties, such as the power dissipation in all the junctions, the current densities, and the charge-carrier mobility, can be calculated from the $V_{i}$ 's and the $G_{i j}^{j u n}$ 's. The complete calculation and analysis is carried out using an in-house developed MATLAB code. 


\section{Limits of the model}

Considering the assumptions and simplifications that we used in our model for charge transport in a SWNT network, there are a few (estimated) limits to the model:

(i) Our model is to be used only at high temperatures $(>90 \mathrm{~K})$. When the thermal energy is similar to or lower than the Coulomb charging energy, equation (2) can no longer be applied as blockade effects become important.

(ii) Our model assumes negligible SWNT segment resistances and thus may not be valid for very long SWNTs with a significant resistance compared to the junction resistances. Therefore, the model should not be applied to very sparse networks with long SWNT segments between the junctions.

(iii) As the junction resistances become smaller with increasing charge carrier density and thus comparable to the segment resistances, the model may not be appropriate anymore.

(iv) For very high areal SWNT densities, the twodimensional box model for the random networks is no longer valid, as the real SWNT film thickness will increase, resulting in an overestimation of the amount of junctions in the model. For such films a three-dimensional Monte Carlo model as presented by Colasanti et al. [34] could be applied for the generation of the network. In that case the computational costs for solving Kirchhoff's equations will increase considerably.

(v) Taking into account only the first van Hove singularity of the DOS might become problematic for networks composed of different SWNT chiralities when the band gaps of the nanotubes differ by more than $\approx 500 \mathrm{meV}$. In that case the second van Hove singularity of the SWNTs with the smaller band gap might become important.

(vi) As we do not know the value of the prefactor $\omega_{0}$ in the Miller-Abrahams hopping rate we cannot calculate the absolute values of the carrier mobilities. We thus only draw conclusions about normalized mobilities and their relative changes with carrier density and other parameters.

(vii) Using a random resistor model to solve the master equation approach is only valid in the approximation for low

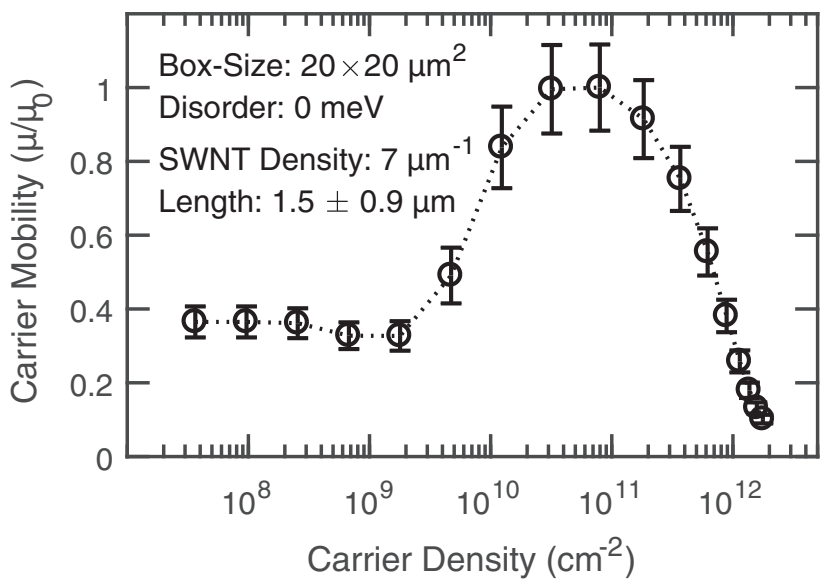

FIG. 3. Normalized mobility versus charge carrier density for a network of $(6,5)$ SWNTs without energetic disorder (simulation box: $20 \times 20 \mu \mathrm{m}^{2}$, SWNT length: $1.5 \pm 0.9 \mu \mathrm{m}$, linear SWNT density: $\left.7 \mu \mathrm{m}^{-1}\right)$. The dotted line is a guide to the eye. electrical fields. Thus, the model is not applicable for high lateral fields.

\section{SIMULATION RESULTS}

In the following, we will present several simulation results on random SWNT networks of a single type of nanotube with different parameters. This will demonstrate the capabilities of our model. One of the major advantages of our model with respect to previous simpler resistor models for carbon nanotubes is its ability to determine the charge carrier density dependence of the mobility. This dependence plays a crucial role in field-effect transistors, where the carrier density is directly tuned by the applied gate voltage. Given the inability of our model to predict absolute mobilities (see previous section), we present all network mobilities normalized to a maximum value $\mu_{0}$. Figure 3 shows representative results for the charge carrier density dependent mobility of a simple random SWNT network, consisting of only $(6,5)$ SWNTs (diameter: $0.757 \mathrm{~nm}$, band gap: $1.27 \mathrm{eV}$ ) without energetic disorder in a $20 \times 20 \mu \mathrm{m}^{2}$ simulation box at room temperature.

For very low carrier densities $\left(<10^{9} \mathrm{~cm}^{-2}\right)$ the mobility is nearly constant, showing a small drop before increasing
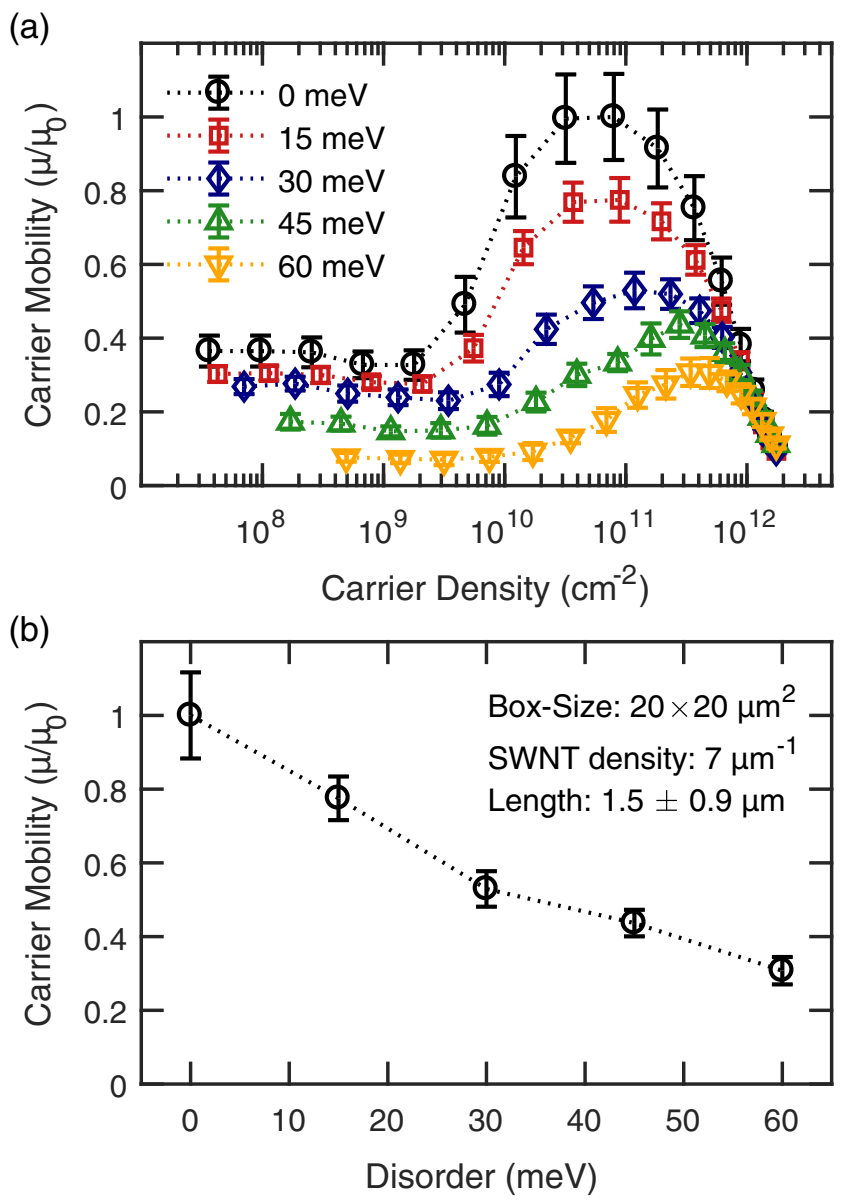

FIG. 4. Network mobilities for different disorder strengths (simulation box size: $20 \times 20 \mu \mathrm{m}^{2}$, SWNT length: $1.5 \pm 0.9 \mu \mathrm{m}$, linear SWNT density: $7 \mu \mathrm{m}^{-1}$ ): (a) Normalized charge carrier mobility versus carrier density. (b) Peak mobility versus disorder strength. Dotted lines are guides to the eye. 
to a maximum value around $10^{11} \mathrm{~cm}^{-2}$. For higher charge carrier densities the mobility drops again due to subband filling, which limits the conductance across the junction at high carrier densities. This effect is exaggerated here because of the absence of higher-lying subbands in the simulation that would start to be filled in a real nanotube network. But overall these results are in good qualitative agreement with experimental data on semiconducting nanotube networks $[3,50]$, which will be discussed in Sec. V.

To obtain the results displayed in Fig. 3, the mobilities of ten networks with different geometries and disorder configurations were calculated and averaged. The standard deviation is represented by the error bars. All of the following results were obtained in this way. For simplicity and ease of comparison of simulation results with different parameters we start by considering networks of only $(6,5)$ SWNTs. Mixtures of SWNTs with different chiralities are investigated in Sec. V B.

\section{A. Influence of energetic disorder}

Experimental data on dense SWNT films reveal inhomogeneous broadening of their spectral features (absorbance and photoluminescence peaks) at room temperature that is about

(a)

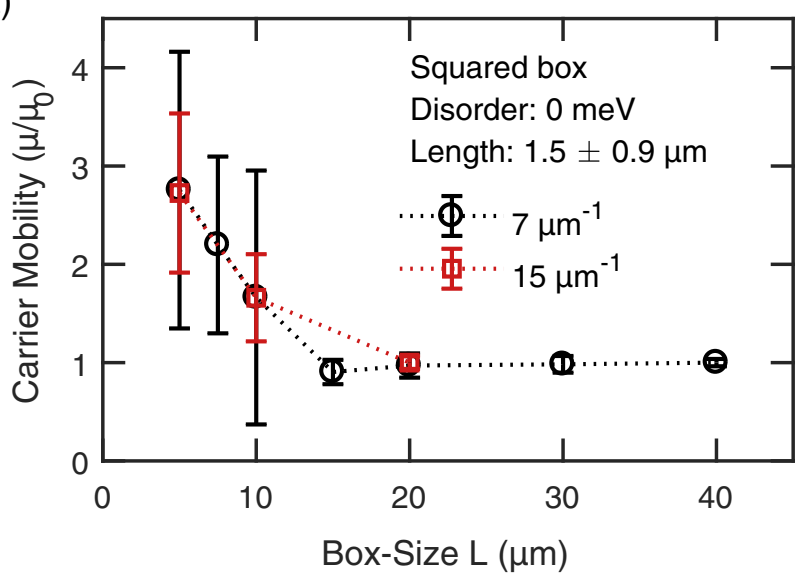

(b)

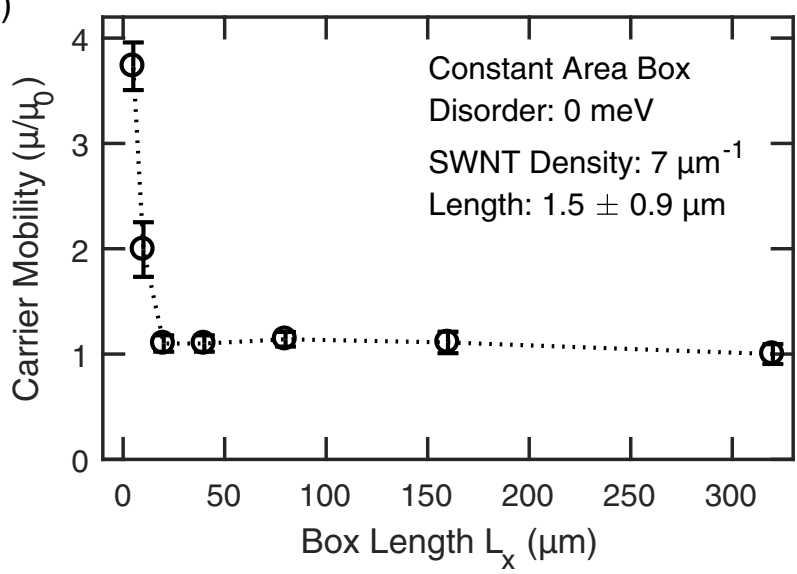

FIG. 5. Network mobilities for different simulation box sizes (energetic disorder: $0 \mathrm{meV}$, SWNT length: $1.5 \pm 0.9 \mu \mathrm{m}$, linear SWNT density $7 \mu \mathrm{m}^{-1}$ and $15 \mu \mathrm{m}^{-1}$ ): (a) Normalized peak mobility for square boxes. (b) Normalized peak mobility for rectangular boxes with constant box area. Dotted lines are guides to the eye.
20 meV larger than for the same SWNTs in dispersion [51,52]. The broadening is likely a result of dipolar disorder in those films. In devices such as field-effect transistors, additional dipolar disorder might be introduced by the substrate and the gate dielectric $[53,54]$. We thus investigate the effect of energetic disorder in this range on the carrier mobility of a random network. Figure 4 shows the simulation results for the carrier density dependent mobility and the maximum mobility for various disorder strengths, defined as the standard deviation of the Gaussian distribution of the shifts in the band edges, ranging from 0 to $60 \mathrm{meV}$.

As generally expected for a disordered system, the carrier mobility of the network drops with increasing disorder (see Fig. 4). The peak mobility decreases almost linearly with disorder strength in the analyzed range. The position of the mobility maximum in Fig. 4(a) shifts to higher charge carrier densities with increasing disorder and the peak shape becomes more asymmetric. For high carrier densities beyond the maximum in the mobility, all curves approach the same low value. Independent of disorder strength, the final limitation for the mobility in a SWNT network is subband filling, as described above.

(a)

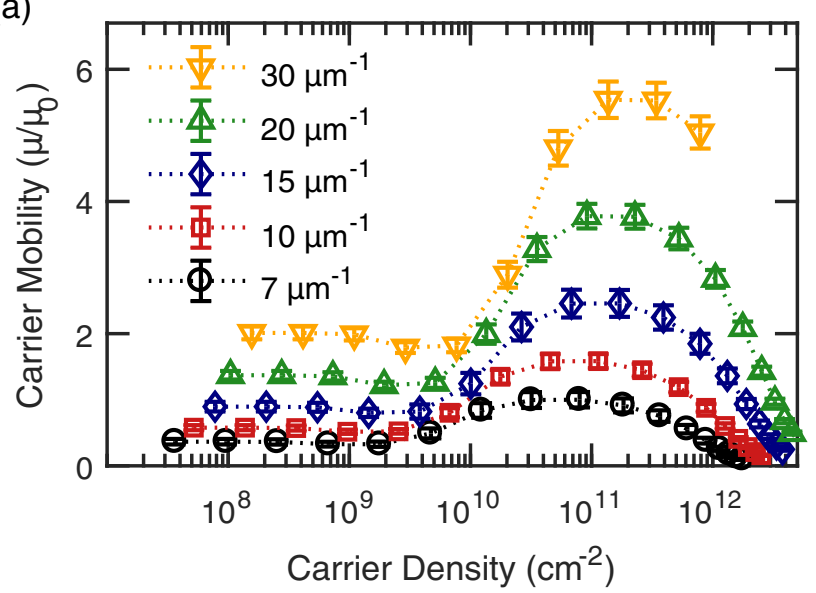

(b)

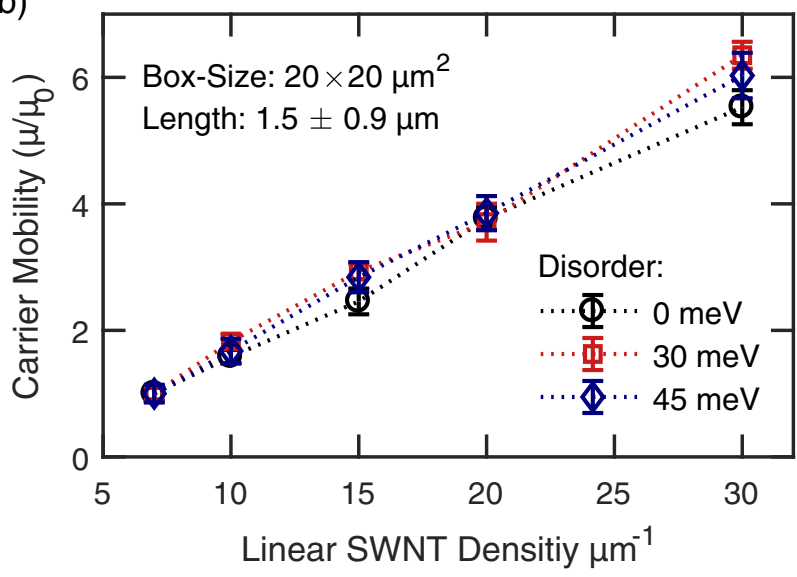

FIG. 6. Network mobilities for different SWNT densities (SWNT length $1.5 \pm 0.9 \mu \mathrm{m}$, simulation box size $20 \times 20 \mu \mathrm{m}^{2}$ ): (a) Normalized mobility versus charge carrier density (without energetic disorder). (b) Peak mobility, normalized to the mobility $\mu_{0}$ for the minimum SWNT density, versus SWNT density for 0, 30, and $45 \mathrm{meV}$ disorder strength. Dotted lines are guides to the eye. 


\section{B. Influence of system size}

We use a stochastic model for the generation of the SWNT network and thus the system size influences the final results obtained for the carrier mobility. In order to determine the minimum system size required for obtaining reliable results we investigated the effect of the size of the simulation box, in conjunction with the linear network density and the length distribution of the nanotubes.

\section{Simulation box size}

Figure 5 shows the dependence of the normalized peak carrier mobility on the simulation box size for square boxes and rectangular boxes with fixed area. Two main effects are evident: First, the standard deviation of the mobilities decreases with increasing box size [see Fig. 5(a)]. A higher linear SWNT density $\left(15 \mu \mathrm{m}^{-1}\right.$ versus $\left.7 \mu \mathrm{m}^{-1}\right)$ also leads to a reduction of the error bars.

Second, the use of shorter simulation boxes in the direction of the electric field for a fixed box area results in a strong increase in mobility [see Fig. 5(b)]. This is because in a very short simulation box a few nanotubes could bridge the entire box. The shorter charge transport paths through the network increase the overall network conductance and mobility. In this regime, the assumption that the SWNT segment resistance is negligible compared to that of the junctions no longer applies.

We find that a box size of around $20 \times 20 \mu \mathrm{m}^{2}$ is sufficient to obtain results with small error bars, while at the same time avoiding an overestimation of the mobilities due to bridging effects. We chose this box size for all simulations presented in the following sections.

\section{Network density}

The SWNT density is an important parameter in device applications. In general, a high density is desired in fieldeffect transistors to reach maximum on-conductances. Figure 6 demonstrates the influence of the SWNT density on charge carrier mobility for our simulations. Here, we chose to vary the linear instead of the areal density, for two reasons:

(i) The experimental determination of the linear SWNT density of a network is straightforward, in contrast to determining the areal density. No information about the SWNT length or its distribution is needed to extract the linear density from atomic force or scanning electron micrographs.

(ii) The linear SWNT density is used to calculate the capacitance of the SWNT network and thus the effective capacitance in a field-effect transistor in order to determine the carrier mobility from current-voltage measurements [55].

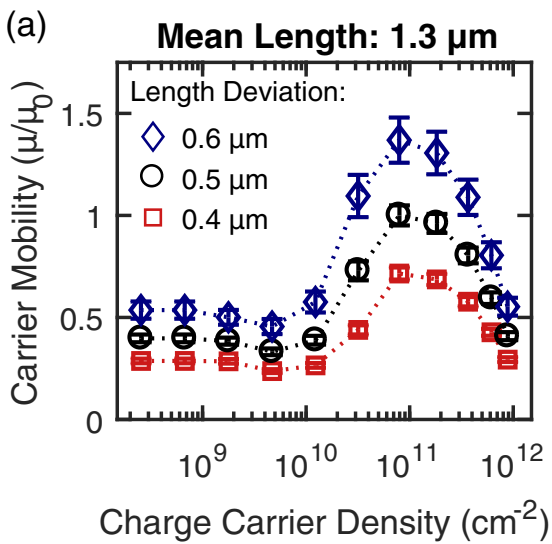

(b)

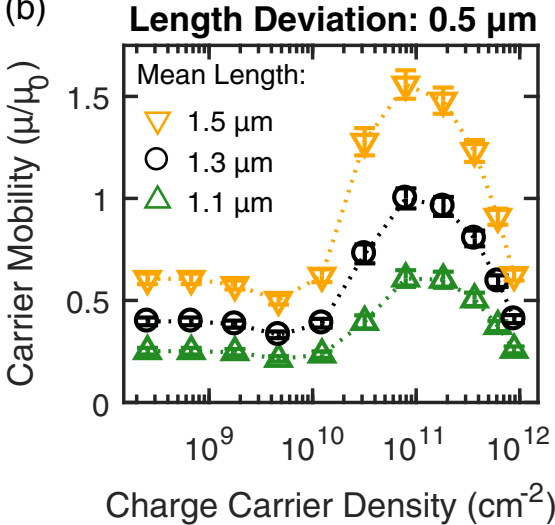

(c)

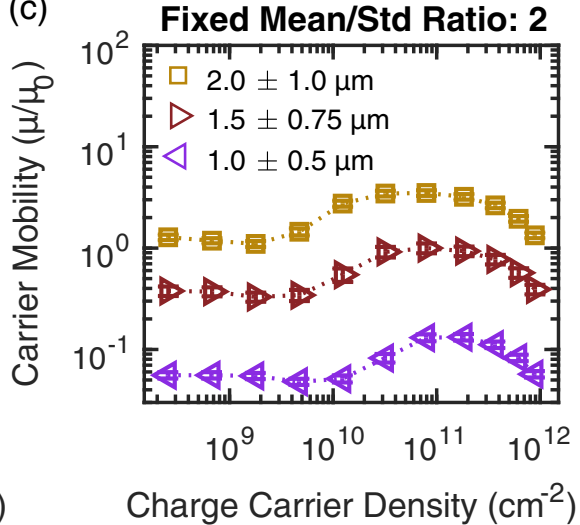

(d)

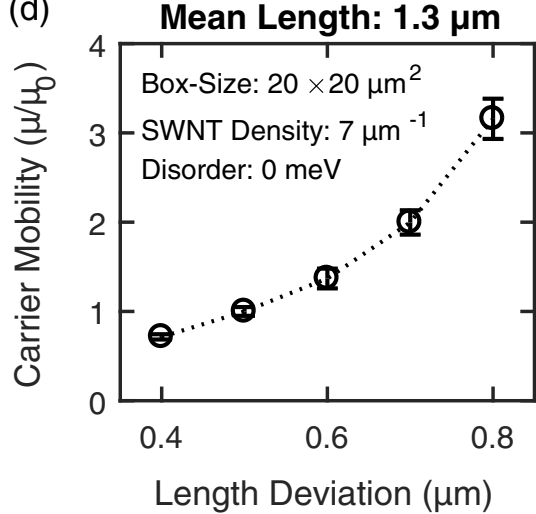

(e)

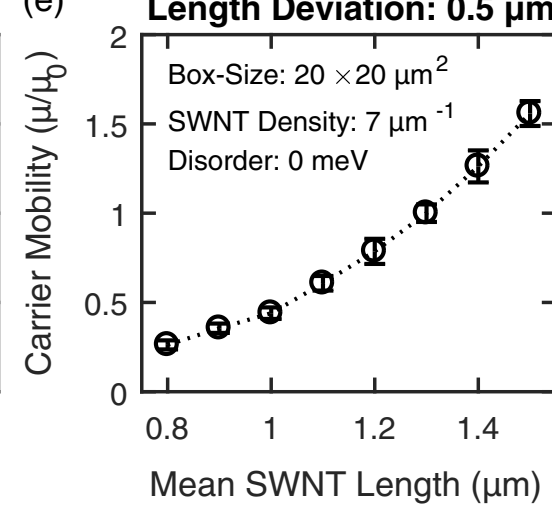

\section{(f)}

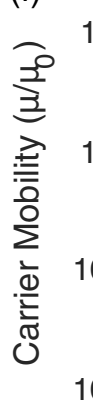

Fixed Mean/Std Ratio: 2

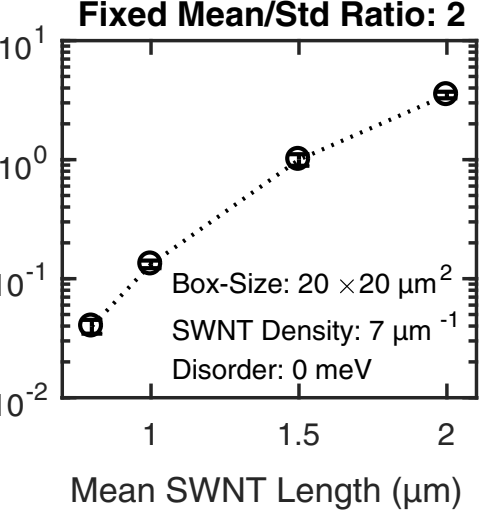

FIG. 7. Normalized carrier mobilities for different SWNT length distributions (no energetic disorder, SWNT density $7 \mu \mathrm{m}^{-1}$, simulation box size $20 \times 20 \mu \mathrm{m}^{2}$ ). Mobility versus charge carrier density and peak mobility versus length standard deviation for fixed SWNT mean length of $1.3 \mu \mathrm{m}$ (a),(d), mobility versus charge carrier density and peak mobility versus mean SWNT length for fixed SWNT length standard deviation of $0.5 \mu \mathrm{m}$ (b),(e), and for fixed ratio between the mean length and length standard deviation of 2 (c),(f). Dotted lines are guides to the eye. 
The comparison of the charge carrier mobility for different SWNT densities reveals two trends. First, the mobility peak position shifts to higher carrier densities for denser networks. Second, the relative peak mobility increases linearly with increasing SWNT density. While the absolute peak mobilities decrease with increasing disorder (see Fig. 4), the relative increase of the peak mobility with increasing SWNT density is independent of the disorder strength. The increasing peak mobility is a simple geometric effect for a two-dimensional network. This should not be the case for a more realistic three-dimensional network [34]. The calculated mobility for high network densities is probably too high, as the amount of junctions and therefore pathways is overestimated. Furthermore, for experimental mobilities in field-effect transistors the limited thickness of the accumulation layer must be considered. Considering the experimental data [21,56,57], we conclude that the presented two-dimensional model should not be applied for SWNT densities larger than 20-30 $\mu \mathrm{m}^{-1}$.

\section{Length distribution}

Different SWNT source materials and dispersion techniques yield different length distributions of SWNTs, which are observed to have a large impact on device performance $[15,18,19]$. Using our model, we can analyze the impact of both average length and length distribution on the network mobility for a constant linear SWNT density (see Fig. 7). For those simulations, we set the energetic disorder to zero, because no specific influence was expected (see Sec. III B 2). We used a gamma distribution as the length distribution function, because it fits the experimental data well [15].

The length dependence of the peak mobility shows the following trends. The broader the length distribution and the longer the nanotubes, the higher is the carrier mobility [see Figs. 7(d)-7(f)], which was expected. However, the dependence in both cases is superlinear. Note that the data for constant mean/standard deviation ratios [Fig. 7(f)] is plotted with a logarithmic vertical axis. Changing both SWNT mean length and standard deviation leads to a substantial change of the charge carrier mobility. Hence, the SWNT length distribution is clearly a critical parameter for device applications that require high mobilities and on-conductances. Similar trends have also been observed experimentally for mixed metallic/semiconducting networks [58]. Interestingly, the carrier density dependence of the mobility [see Figs. 7(a)-7(c)] reveals a trend that is opposed to the trend observed for different linear SWNT densities. The peak mobilities shift to slightly lower carrier densities for broader distributions and longer nanotubes as the areal SWNT density decreases for longer tubes at constant linear density.

\section{VISUALIZATION OF CURRENT PATHS}

The previous examples have shown that our model can reproduce the typically expected and observed correlations
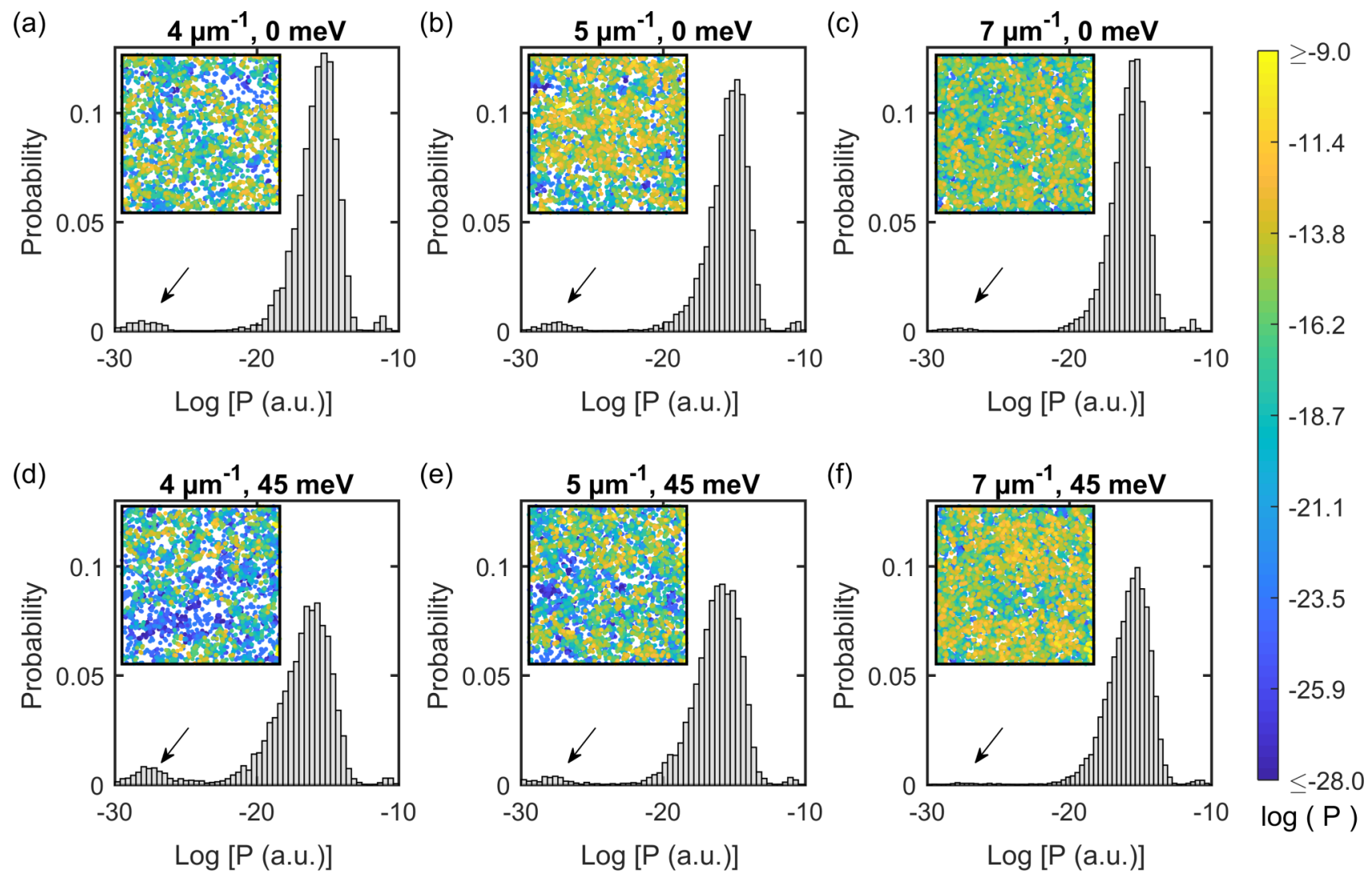

FIG. 8. Histograms of the junction power dissipation $(P)$ distribution (with color-coded visualization as insets) for different network densities and energetic disorder strengths (see subfigure titles). The SWNT length distribution is $1.1 \pm 0.5 \mu \mathrm{m}$. The carrier densities are those at the peak mobilities $\left(n_{a}=1.0 \times 10^{11} \mathrm{~cm}^{-2}, n_{b}=1.3 \times 10^{11} \mathrm{~cm}^{-2}, n_{c}=1.8 \times 10^{11} \mathrm{~cm}^{-2}, n_{d}=2.5 \times 10^{11} \mathrm{~cm}^{-2}, n_{e}=3.1 \times 10^{11} \mathrm{~cm}^{-2}\right.$, and $n_{f}=4.5 \times 10^{11} \mathrm{~cm}^{-2}$ ). 
(a)

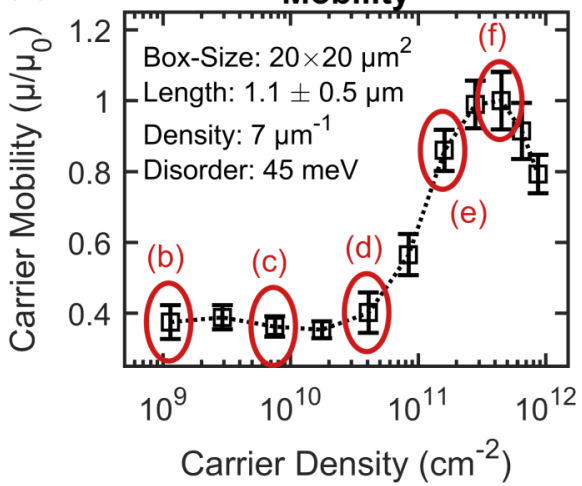

(d)

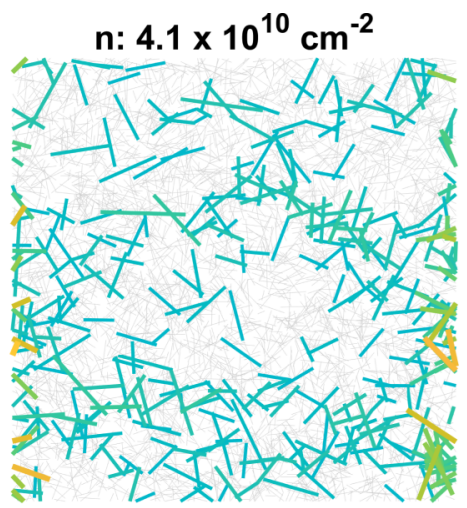

(b)

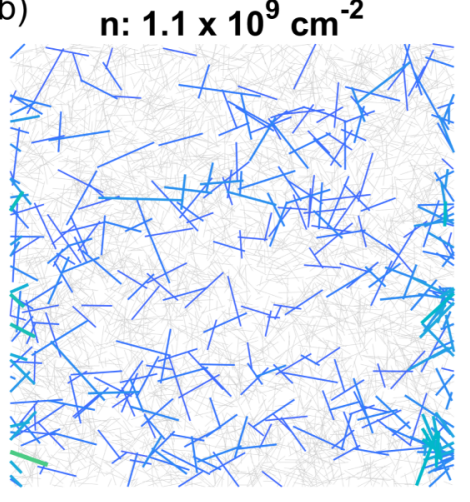

(e)

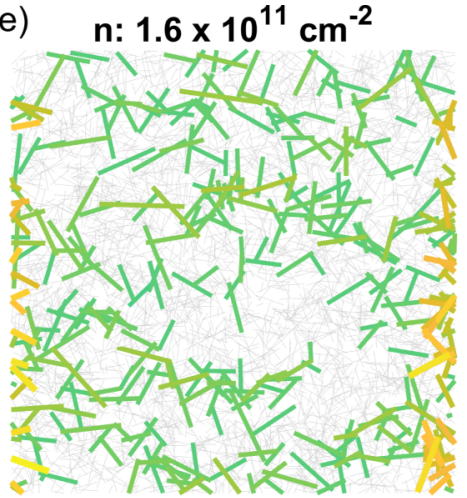

(c)

(c) $\mathrm{n}: 7.6 \times 10^{9} \mathrm{~cm}^{-2}$
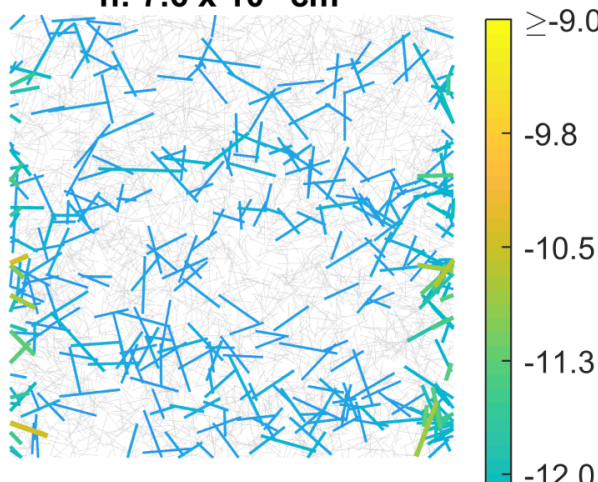

(f)

f) $4.5 \times 10^{11} \mathrm{~cm}^{-2}$

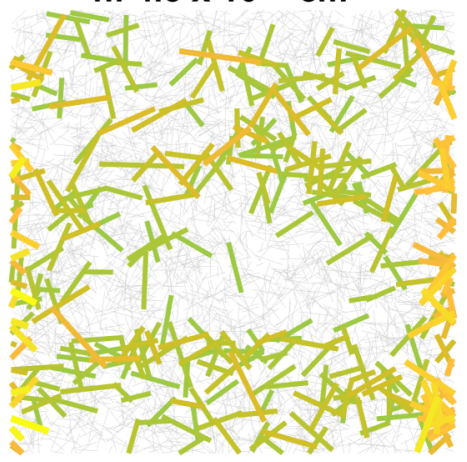

FIG. 9. (a) Normalized charge carrier mobility versus charge carrier density. The dotted line is a guide to the eye. (b)-(f) Visualization of current paths through a network with $45 \mathrm{meV}$ energetic disorder at different charge carrier densities $n$ as labeled in (a). The 10\% of SWNTs that carry the most current in the network have been colored (other SWNTs are light gray) and given a thickness proportional to the logarithm of the current (in a.u.) they carry. Simulation box sizes are $20 \times 20 \mu \mathrm{m}^{2}$.

between SWNT network mobilities and basic network parameters. But the model also allows us to visualize current paths and power dissipation within a network and to identify bottlenecks for charge transport. Figure 8 shows histograms and maps of the power dissipation in the junctions of SWNT networks with different densities and energetic disorder strengths. Again, for simplicity we analyze only networks of $(6,5)$ SWNTs and we apply a fixed length distribution of $1.1 \pm 0.5 \mu \mathrm{m}$.

Similar to other percolating resistor networks, most of the power is dissipated in only a few resistors, i.e. tube-tube junctions. Note that the horizontal power $(P)$ axis in the histograms is logarithmic. Figures 8 (a)-8(c) visualize the statistics of the power dissipation in $(6,5)$ SWNT networks without energetic disorder at low nanotube densities (4-7 $\mu \mathrm{m}^{-1}$ ), close to the percolation threshold. The carrier densities are those at which the mobility peaks. We observe that the power dissipation becomes more homogeneous for denser networks. With increasing network density we are moving away from the percolation threshold, so that the network becomes more interconnected and the fraction of junctions in which almost no power is dissipated decreases (see marked fraction in Fig. 8). At the same time, the fraction of junctions in which most of the power is dissipated hardly changes (for details see below).

The introduction of energetic disorder of $45 \mathrm{meV}$ [see Figs. 8(d)-8(f)] makes these trends more pronounced. The power dissipation distribution becomes broader, i.e., more inhomogeneous. In particular, the number of junctions with low power dissipation for the case of the low-density network
(4 $\mu \mathrm{m})$ is much larger. Energetic disorder creates network areas that carry only a small part of the current, with a clear effect on the overall network mobility, as shown in Figs. 4(a) and $4(\mathrm{~b})$.

In addition to visualizing the power dissipation distribution in the junctions, we can also visualize the distribution of

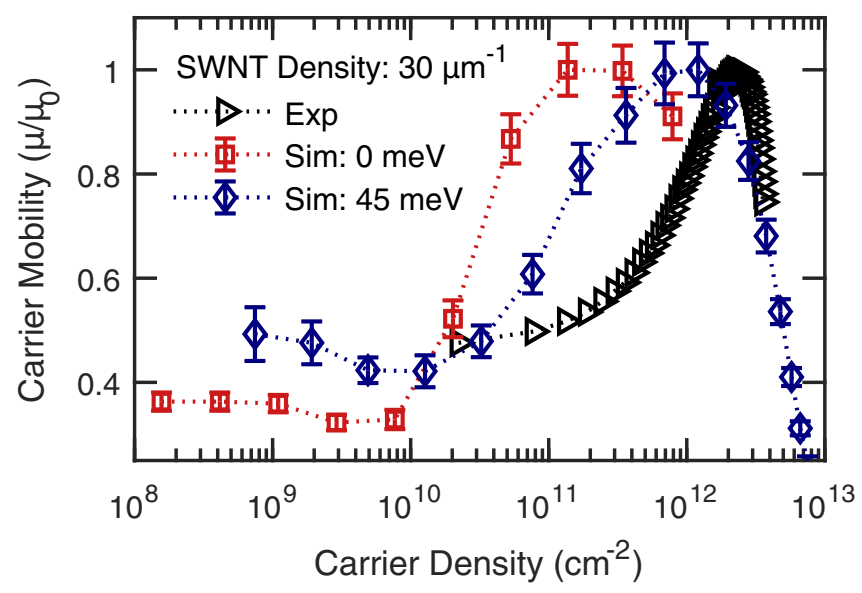

FIG. 10. Experimental [52] and simulated normalized mobility versus charge carrier density for $(6,5)$ SWNT networks. Simulated results are given for the cases without energetic disorder and an energy disorder of $45 \mathrm{meV}$. The SWNT density was estimated from the experiments to be $30 \mu \mathrm{m}^{-1}$. Dotted lines are guides to the eye. 


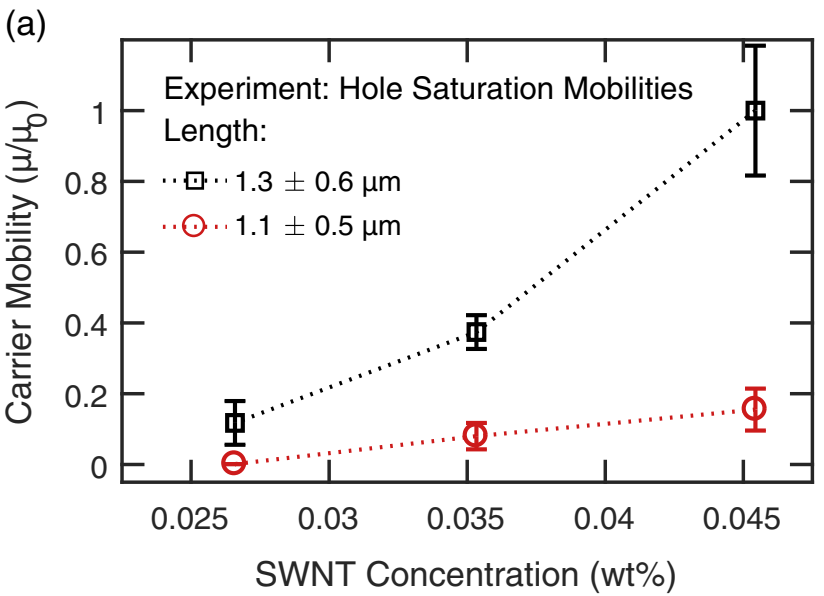

(b)

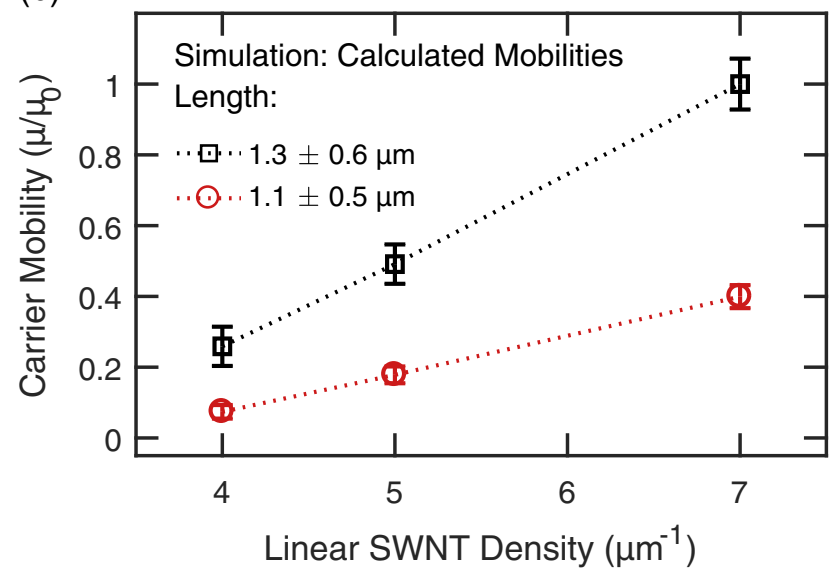

FIG. 11. Experimental [20] (a) and simulated (b) peak mobilities (energetic disorder: $45 \mathrm{meV}$, simulation box size $20 \times 20 \mu \mathrm{m}^{2}$ ) for two different SWNT length distributions of a $(6,5)$ SWNT network. Dotted lines are guides to the eye.

current among the SWNTs themselves. By this means we can identify the current pathways through the network. Figures 9(b)-9(f) show, for an energy disorder of $45 \mathrm{meV}$ and at different carrier densities, as indicated in Fig. 9(a), color coded representations of those SWNTs in the network that carry most of the current. Note that, due to the applied periodic boundary conditions, the current (and thus also the power dissipation) at the edges of the box (in the field direction) is fixed and therefore larger than in the rest of the network. Figures 9(b)-9(f) clearly indicate that most of the current is actually carried by only a fraction of SWNTs within the network forming low-resistance pathways. This effect was also observed experimentally [20,22]. The current pathways slightly change with increasing charge carrier density. This change is caused by SWNTs with a band edge that is shifted to higher energy by the energy disorder, so that they only become accessible at high carrier densities and subsequently change the map of current pathways.

\section{COMPARISON TO EXPERIMENT}

In order to verify the practical applicability of the above established model, we now apply it to experimental SWNT networks and compare the results to available charge transport data. We start with a qualitative and quantitative comparison of modeled and measured charge carrier dependent mobilities of networks of a single chirality of nanotubes [20,52], and then turn to a mixed network with different chiralities [22].

\section{A. Networks of a single SWNT type}

As an experimental test system we again choose $(6,5)$ SWNTs. They can be produced and dispersed in large amounts and with high chiral purity by polymer wrapping [15]. We compare experimental results of aerosol jet printed $(6,5)$ SWNT networks in field-effect transistors [52] to our simulation of dense networks $\left(30 \mu \mathrm{m}^{-1}\right)$. The experimental charge carrier density dependent mobility was extracted from the transconductance of the channel using the measured on-state capacitance. The charge carrier density was estimated from the on-state capacitance multiplied by the gate overdrive.

Figure 10 shows the normalized carrier density dependent mobilities for the aerosol jet printed SWNTs together with simulated results. The experimental data show the previously

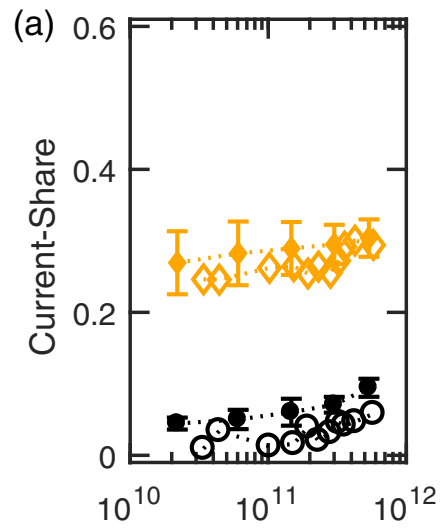

Carrier Density $\left(\mathrm{cm}^{-2}\right)$

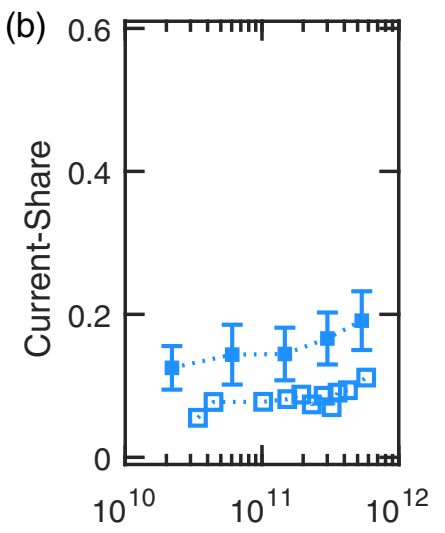

Carrier Density $\left(\mathrm{cm}^{-2}\right)$

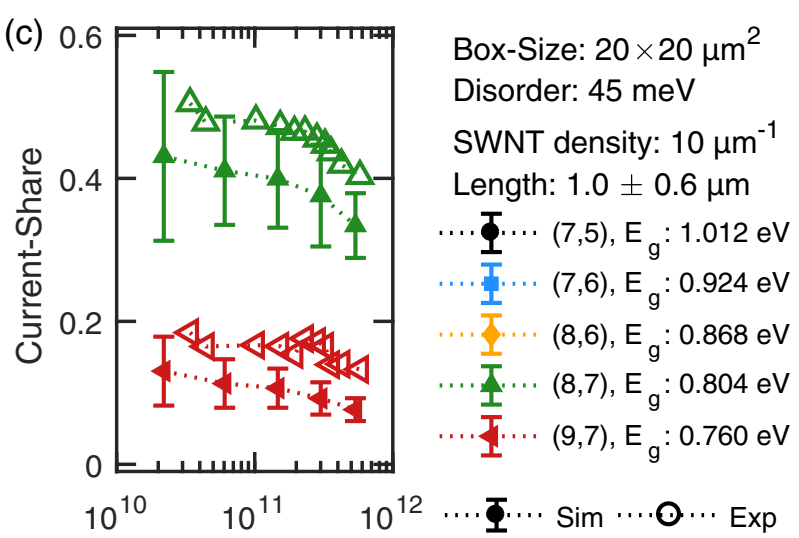

Carrier Density $\left(\mathrm{cm}^{-2}\right)$

FIG. 12. Comparison of experimental [22] (open symbols) to simulated (closed symbols with error bars) results for the share in the current of each nanotube species in a mixed network of five chiralities: (a) $(7,5)$ and $(8,6),(b)(7,6)$, and (c) $(8,7),(9,7)$. Dotted lines are guides to the eye. 
reported mobility maximum $[3,50]$, which occurs due to band filling of the first subband, at carrier densities of around $2-3 \times 10^{12} \mathrm{~cm}^{-2}$. A comparable behavior is found for the simulated mobilities. By introducing energetic disorder, the peak position shifts toward the peak in the experimental data. The presence of energetic disorder as well as shallow traps can be expected due to the substrate, the dielectric, or impurities. Overall, the results are in good qualitative, but not quantitative agreement. A possible reason for the quantitative mismatch might be an incorrect estimate of the SWNT density. As the networks resulting from aerosol jet printed devices are rather thick, the density of around $30 \mu \mathrm{m}^{-1}$ used in the simulations might underestimate the experimental value. Using higher SWNT densities would lead to a shift of the mobility maximum to higher carrier densities, as shown in Sec. III. However, at such high densities the model may become unreliable, as the film can no longer be treated as a two-dimensional network (see Sec. II C).

Next, we compare experimental data for the dependence of the mobility on the mean nanotube length and the length distribution to the predictions of our model. Here we use data by Malhofer et al. [20] on $(6,5)$ SWNT networks with different mean lengths embedded in an insulating polymer matrix. Experimentally, small changes in the SWNT length distribution $(1.1 \pm 0.5 \mu \mathrm{m}$ vs $1.3 \pm 0.6 \mu \mathrm{m})$ were found to have a strong impact on the effective mobility, which we try to reproduce with our model.
Figure 11 shows the experimental (a) and simulated (b) dependence of the carrier mobility on the density of the SWNT network for two different length distributions, based on the given network parameters. The network density in the simulations was increased according to the experimental concentration of SNWTs in the polymer matrix given in weight percent. With increasing SWNT concentration (density) and length, the mobility increases, as expected. Both the experimental and simulated data show that the SWNT length distribution has an unexpectedly large impact on the charge carrier mobility. A variation in the length distribution by only $18 \%$ (a few hundred nanometers) alters the mobility by a factor of 2-3. The impact of this variation even increases for higher SWNT densities. As shown in Fig. 7(d), a broader length distribution with a few longer nanotubes can significantly change the network mobility. These results demonstrate that our model is able to qualitatively describe the charge transport in networks of a single type of nanotubes.

\section{B. Mixed networks}

The most important motivation for the development of the presented model was the description of charge transport in carbon nanotube networks that contain many different species of semiconducting SWNTs. Different chiralities exhibit different diameters, band gaps, and DOS. These mixed networks are of special interest, as they are most commonly used in devices. In

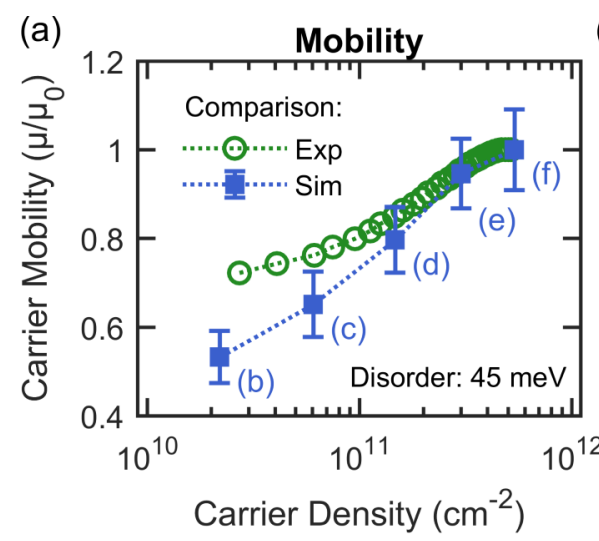

(b)

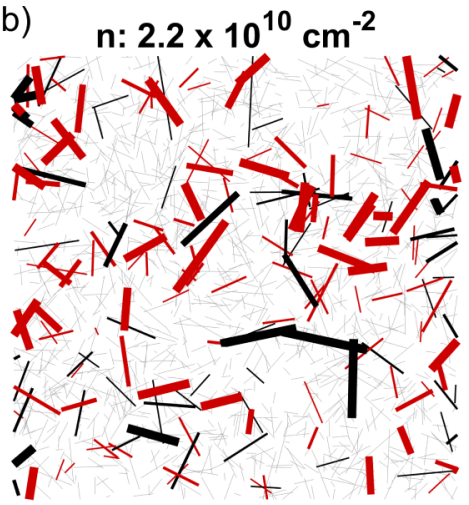

(d)

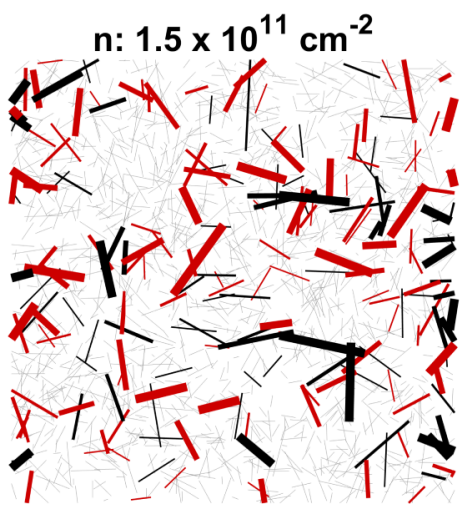

(e)

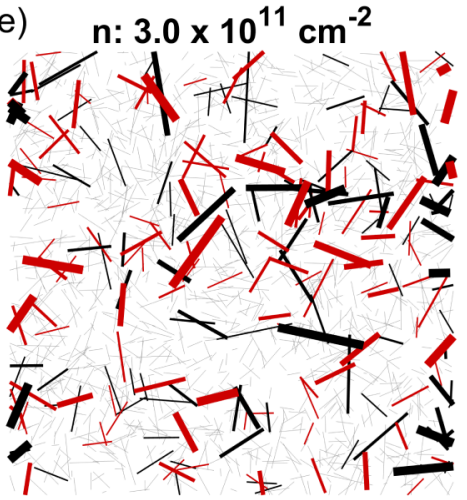

(c) $\mathrm{n}: 6.1 \times 10^{10} \mathrm{~cm}^{-2}$
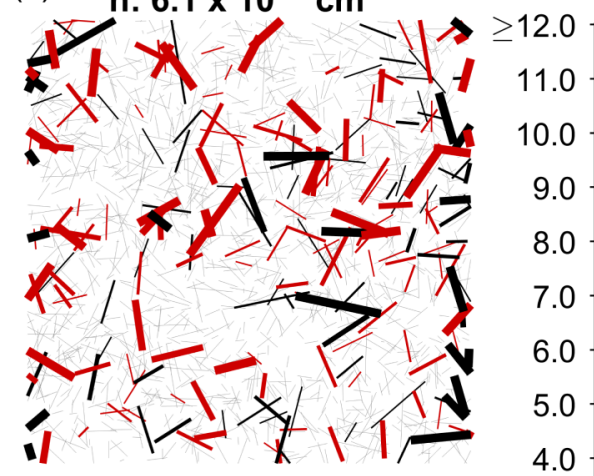

(f)

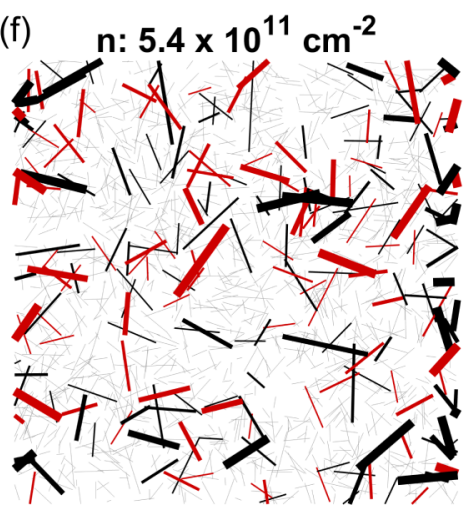

3.0

2.0

2.0

1.0

$\mathrm{I}_{\mathrm{i}} / \mathrm{I}_{\mathrm{avg}}$

$(9,7)$

$(n, m)$

FIG. 13. (a) Comparison of experimental [22] to simulated carrier density dependence of the mobility in a mixed network of five chiralities (density: $10 \mu \mathrm{m}^{-1}$, length distribution: $1.0 \pm 0.5 \mu \mathrm{m}$ ). Dotted lines are guides to the eye. (b)-(f) Simulated current maps at the carrier densities indicated in (a), with nanotubes that carry more than the average current per tube highlighted [red: $(9,7)$ SWNTs, black: (7,5) SWNTs]. Gray lines represent SWNTs that carry less than the average current. The thickness of each drawn nanotube is proportional to the relative current $I_{i} / I_{\text {avg }}$ it carries. 
order to understand the impact of the network composition on device performance, it is necessary to include different SWNT chiralities, with their conduction band edges and DOS in the simulations.

As a proof of concept we investigate a particular network containing five different nanotube species and compare the simulation results to previous experimental results obtained for these networks [22]. The five nanotube chiralities are $(7,5)(19 \%$, diameter $0.83 \mathrm{~nm}$, band gap $1.21 \mathrm{eV}),(7,6)(21 \%$, diameter $0.89 \mathrm{~nm}$, band gap $1.11 \mathrm{eV}),(8,6)(28 \%$, diameter $0.97 \mathrm{~nm}$, band gap $1.06 \mathrm{eV}),(8,7)(26 \%$, diameter $1.03 \mathrm{~nm}$, band gap $0.98 \mathrm{eV})$, and $(9,7)(6 \%$, diameter $1.10 \mathrm{~nm}$, band gap $0.94 \mathrm{eV})$. The experimental network composition was determined from absorption measurements using established absorption cross sections for each SWNT species. The current distribution within the network was deduced from electroluminescence spectra recorded at different gate voltages (carrier densities) from light-emitting field-effect transistors based on these networks, taking into account the photoluminescence yield of the different nanotube species and energy transfer within the network. For further experimental details we refer to Rother et al. [22]. The experimentally determined share in the current carried by each nanotube species as a function of carrier density is plotted in Fig. 12 (open symbols). Note that the current share is not proportional to the abundance of each nanotube species in the network. For example, the $(9,7)$ nanotubes that make up only $6 \%$ of the network carry more than $40 \%$ of the current. As the charge carrier density increases, the nanotubes with larger band gaps start to contribute more to the charge transport [see Figs. 12(a)-12(b)], while the current-share of chiralities with a smaller band gap decreases [see Fig. 12(c)].

We performed simulation for this network with the experimentally determined network properties, assuming an energetic disorder of $45 \mathrm{meV}$, and calculated the current share of each nanotube species, as shown in Fig. 12 (filled symbols with error bars). The disorder strength and the charge carrier densities are the only free parameters in the simulations. All other parameters are given by the experiment. The experimentally determined charge carrier densities might deviate somewhat from their true values due to assumptions about the effective device capacitances, charge accumulation behavior, and threshold voltages. The experimental and simulated values of the current shares and their dependence on carrier density agree remarkably well. In addition to the current shares, we find a reasonably good agreement for the carrier density dependent mobility [see Fig. 13(a)]. Hence, our model is able to describe the charge transport properties of realistic networks containing several SWNT species quite well.

In our previous work we could only qualitatively describe the experimental data, using an equilibrium charge accumulation model [22], which lacked the ability to account for the specific current pathways followed by the mobile charges. With the present model we have overcome this limitation and can now quantitatively describe the steady state current distribution and visualize it for different carrier densities. Figures 13(b)-13(f) show the currents carried by two selected chiralities within the network, namely the $(7,5)$ SWNT (largest band gap) and the $(9,7)$ SWNTs (smallest band gap). Only nanotubes that carry a current larger than the average current per nanotube are highlighted and the line thickness scales with the current carried by a nanotube. For the lowest carrier density [Fig. 13(b)] the $(9,7)$ nanotubes (red) dominate the charge transport, although their abundance in the network is only $6 \%$. For increasing charge carrier density [Figs. 13(c)-13(f)], the relative current carried by the $(9,7)$ tubes decreases, while an increasing number of $(7,5)$ SWNTs (black) contribute to the current. Hence, the presented charge transport model not only reproduces the experimental observations for mixed SWNT networks well but also enables deeper insights into the charge transport pathways and current distribution in such complex networks.

\section{CONCLUSION}

We presented a model for the simulation of charge transport in two-dimensional semiconducting single-walled carbon nanotube (SWNT) networks that are treated as a random resistor network of SWNT-SWNT junctions. We neglected the resistance of the SWNT segments and modified the conventional random-resistor network approach for disordered systems by including the density of states (DOS) of the individual SWNTs. The model enables simulation of charge carrier density dependent mobilities for networks of a single nanotube chirality as well as networks containing nanotubes with different diameters and band gaps, with the additional possibility to include energetic disorder. The simulated charge carrier density dependencies of the network mobility are in good agreement with experimental data on networks of SWNTs with different length distributions and on mixed networks of different nanotube chiralities. In mixed networks the simulated shares in the current carried by different nanotube species and their carrier density dependence agree remarkably well with the experiment. Furthermore, the simulations enable visualization of power dissipation and current distribution within simple and complex networks. The developed model is a powerful tool to design and investigate semiconducting SWNT networks, taking into account network parameters such as the nanotube density, the chirality composition of the network, and the length distribution of the nanotubes. Applying this model should facilitate rational prediction and improvement of the performance of practical nanotube network devices like field-effect transistors.

\section{ACKNOWLEDGMENTS}

This research was funded by European Research Council under the European Union's Seventh Framework Programme (FP/2007-2013)/ERC Grant Agreement No. 306298 (ENLUMINATE). S.P.S. thanks the Graduate School of Advanced Materials and Processes (GS-AMP, Erlangen), for financial support. J.Z. thanks the Alfried Krupp von Bohlen und Halbach-Stiftung via the Alfried Krupp Förderpreis für junge Hochschullehrer for general support. A.M. and P.A.B. acknowledge support by the Dutch Technology Foundation $S T W$, the applied science division of NWO, and the Technology Program of the Dutch Ministry of Economic Affairs (project No. 12200). X.dV. and P.A.B. acknowledge support by the EU through the Horizon 2020 EU project MOSTOPHOS (Contract No. 646259). 
[1] V. Derenskyi, W. Gomulya, J. M. S. Rios, M. Fritsch, N. Fröhlich, S. Jung, S. Allard, S. Z. Bisri, P. Gordiichuk, A. Herrmann, U. Scherf, and M. A. Loi, Adv. Mater. 26, 5969 (2014).

[2] G. J. Brady, Y. Joo, S. Singha Roy, P. Gopalan, and M. S. Arnold, Appl. Phys. Lett. 104, 083107 (2014).

[3] S. P. Schieß1, N. Fröhlich, M. Held, F. Gannott, M. Schweiger, M. Forster, U. Scherf, and J. Zaumseil, ACS Appl. Mater. Interfaces 7, 682 (2015).

[4] S. G. Bucella, J. M. Salazar-Rios, V. Derenskyi, M. Fritsch, U. Scherf, M. A. Loi, and M. Caironi, Adv. Electron. Mater. 2, 1600094 (2016).

[5] B. Chen, P. Zhang, L. Ding, J. Han, S. Qiu, Q. Li, Z. Zhang, and L. M. Peng, Nano Lett. 16, 5120 (2016).

[6] D.-M. Sun, M. Y. Timmermans, A. Kaskela, A. G. Nasibulin, S. Kishimoto, T. Mizutani, E. I. Kauppinen, and Y. Ohno, Nat. Commun. 4, 2302 (2013).

[7] F. Xu, M. Y. Wu, N. S. Safron, S. S. Roy, R. M. Jacobberger, D. J. Bindl, J. H. Seo, T. H. Chang, Z. Ma, and M. S. Arnold, Nano Lett. 14, 682 (2014).

[8] D. M. Sun, C. Liu, W. C. Ren, and H. M. Cheng, Small 9, 1188 (2013).

[9] J. Zaumseil, Semicond. Sci. Technol. 30, 074001 (2015).

[10] J. C. Charlier, X. Blase, and S. Roche, Rev. Mod. Phys. 79, 677 (2007).

[11] M. S. Arnold, A. A. Green, J. F. Hulvat, S. I. Stupp, and M. C. Hersam, Nat. Nanotechnol. 1, 60 (2006).

[12] A. Nish, J.-Y. Hwang, J. Doig, and R. J. Nicholas, Nat. Nanotechnol. 2, 640 (2007).

[13] H. Liu, D. Nishide, T. Tanaka, and H. Kataura, Nat. Commun. 2, 309 (2011).

[14] J. A. Fagan, C. Y. Khripin, C. A. Silvera Batista, J. R. Simpson, E. H. Hároz, A. R. Hight Walker, and M. Zheng, Adv. Mater. 26, 2800 (2014).

[15] A. Graf, Y. Zakharko, S. P. Schieß1, C. Backes, M. Pfohl, B. S. Flavel, and J. Zaumseil, Carbon 105, 593 (2016).

[16] W. Gomulya, G. D. Costanzo, E. J. F. De Carvalho, S. Z. Bisri, V. Derenskyi, M. Fritsch, N. Fröhlich, S. Allard, P. Gordiichuk, A. Herrmann, S. J. Marrink, M. C. Dos Santos, U. Scherf, and M. A. Loi, Adv. Mater. 25, 2948 (2013).

[17] G. J. Brady, A. J. Way, N. S. Safron, H. T. Evensen, P. Gopalan, and M. S. Arnold, Sci. Adv. 2, e1601240 (2016).

[18] Y. Kuwahara, F. Nihey, S. Ohmori, and T. Saito, Carbon 91, 370 (2015).

[19] H. Gui, H. Chen, C. Y. Khripin, B. Liu, J. A. Fagan, C. Zhou, and M. Zheng, Nanoscale 8, 3467 (2016).

[20] A. Malhofer, M. Rother, Y. Zakharko, A. Graf, S. P. Schieß1, and J. Zaumseil, Org. Electron. 45, 151 (2017).

[21] V. K. Sangwan, R. P. Ortiz, J. M. P. Alaboson, J. D. Emery, M. J. Bedzyk, L. J. Lauhon, T. J. Marks, and M. C. Hersam, ACS Nano 6, 7480 (2012).

[22] M. Rother, S. P. Schieß1, Y. Zakharko, F. Gannott, and J. Zaumseil, ACS Appl. Mater. Interfaces 8, 5571 (2016).

[23] K. Yanagi, H. Udoguchi, S. Sagitani, Y. Oshima, T. Takenobu, H. Kataura, T. Ishida, K. Matsuda, and Y. Maniwa, ACS Nano 4, 4027 (2010).

[24] J. Gao and Y.-L. L. Loo, Adv. Funct. Mater. 25, 105 (2015).
[25] M. E. Itkis, A. Pekker, X. Tian, E. Bekyarova, and R. C. Haddon, Acc. Chem. Res. 48, 2270 (2015).

[26] S. Kumar, J. Y. Murthy, and M. A. Alam, Phys. Rev. Lett. 95, 066802 (2005).

[27] A. Behnam and A. Ural, Phys. Rev. B 75, 125432 (2007).

[28] J. Hicks, A. Behnam, and A. Ural, Phys. Rev. E 79, 012102 (2009).

[29] M. A. Topinka, M. W. Rowell, D. Goldhaber-Gordon, M. D. McGehee, D. S. Hecht, and G. Gruner, Nano Lett. 9, 1866 (2009).

[30] L. F. C. Pereira, C. G. Rocha, A. Latgé, and M. S. Ferreira, J. Appl. Phys. 108, 103720 (2010).

[31] P. S. Kang and G. T. Kim, Phys. Status Solidi (b) 248, 2644 (2011).

[32] E. Albert, A. Abdellah, G. Scarpa, and P. Lugli, in Proceedings of the 2012 12th IEEE International Conference on Nanotechnology (IEEE-NANO) (IEEE, Piscataway, NJ, 2012), pp. 1-4.

[33] L. Wen and M. Zhang, in Proceedings of the 2016 IEEE 16th International Conference on Nanotechnology (IEEE-NANO) (IEEE, Piscataway, NJ, 2016), pp. 959-962.

[34] S. Colasanti, V. D. Bhatt, A. Abdelhalim, and P. Lugli, IEEE Trans. Electron Devices 63, 1346 (2016).

[35] M. S. Fuhrer, J. Nygård, L. Shih, M. Forero, Y.-G. Yoon, M. S. C. Mazzoni, H. J. Choi, J. Ihm, S. G. Louie, A. Zettl, and P. L. McEuen, Science 288, 494 (2000).

[36] A. Javey, J. Guo, Q. Wang, M. Lundstrom, and H. Dai, Nature (London) 424, 654 (2003).

[37] F. Bottacchi, S. Bottacchi, F. Späth, I. Namal, T. Hertel, and T. D. Anthopoulos, Small 12, 4211 (2016).

[38] M. Stadermann, S. J. Papadakis, M. R. Falvo, J. Novak, E. Snow, Q. Fu, J. Liu, Y. Fridman, J. J. Boland, R. Superfine, and S. Washburn, Phys. Rev. B 69, 201402 (2004).

[39] P. N. Nirmalraj, P. E. Lyons, S. De, J. N. Coleman, and J. J. Boland, Nano Lett. 9, 3890 (2009).

[40] A. Znidarsic, A. Kaskela, P. Laiho, M. Gaberscek, Y. Ohno, A. G. Nasibulin, E. I. Kauppinen, and A. Hassanien, J. Phys. Chem. C 117, 13324 (2013).

[41] A. Miller and E. Abrahams, Phys. Rev. 120, 745 (1960).

[42] V. Ambegaokar, B. I. Halperin, and J. S. Langer, Phys. Rev. B 4, 2612 (1971).

[43] M. C. J. M. Vissenberg and M. Matters, Phys. Rev. B 57, 12964 (1998).

[44] J. Cottaar, L. J. A. Koster, R. Coehoorn, and P. A. Bobbert, Phys. Rev. Lett. 107, 136601 (2011).

[45] X. Zhou, J.-Y. Park, S. Huang, J. Liu, and P. L. McEuen, Phys. Rev. Lett. 95, 146805 (2005).

[46] S. J. Tans, M. H. Devoret, H. J. Dai, A. Thess, R. E. Smalley, L. J. Geerligs, and C. Dekker, Nature (London) 386, 474 (1997).

[47] R. Saito, G. Dresselhaus, and M. S. Dresselhaus, Phys. Rev. B 61, 2981 (2000).

[48] S. Maruyama, 1D DOS (van Hove singularity), http:// www.photon.t.u-tokyo.ac.jp/ maruyama/kataura/1D_DOS.html (2002).

[49] MATLAB 2016a (Mathworks(R), US).

[50] H. Shimotani, S. Tsuda, H. Yuan, Y. Yomogida, R. Moriya, T. Takenobu, K. Yanagi, and Y. Iwasa, Adv. Funct. Mater. 24, 3305 (2014). 
[51] A. Graf, L. Tropf, Y. Zakharko, J. Zaumseil, and M. C. Gather, Nat. Commun. 7, 13078 (2016).

[52] M. Rother, M. Brohmann, S. Yang, S. B. Grimm, S. P. Schieß1, A. Graf, and J. Zaumseil, Adv. Electron. Mater. 3, 1700080 (2017).

[53] S. H. Lee, Y. Xu, D. Khim, W. T. Park, D. Y. Kim, and Y. Y. Noh, ACS Appl. Mater. Interfaces 8, 32421 (2016).

[54] M. Held, S. P. Schieß1, D. Miehler, F. Gannott, and J. Zaumseil, Appl. Phys. Lett. 107, 083301 (2015).
[55] Q. Cao, M. Xia, C. Kocabas, M. Shim, J. A. Rogers, and S. V. Rotkin, Appl. Phys. Lett. 90, 023516 (2007).

[56] Z. Li, J. Ding, J. Lefebvre, and P. R. Malenfant, Org. Electron. 26, 15 (2015)

[57] S. P. Schieß1, F. Gannott, S. H. Etschel, M. Schweiger, S. Grünler, M. Halik, and J. Zaumseil, Adv. Mater. Interfaces 3, 1600215 (2016).

[58] D. Hecht, L. Hu, and G. Grüner, Appl. Phys. Lett. 89, 133112 (2006). 